\title{
A Study on the Future of Rural Dwellings
}

\author{
Zhu Yali \\ Environmental Art Design Department \\ Hubei Academy of Fine Arts \\ 24672405@qq.com
}

\begin{abstract}
In the context of the large-scale new rural construction in China, this paper attempts to analyze the current residential construction behavior from the perspective of future. Through the comparison of the houses of different periods left in this stage and the present houses, according to the development of living habits, this paper analyzes what kind of residential buildings will be preserved, why will they be preserved and what will these preserved houses bring to future people, so as to explore how to build the current rural residential.
\end{abstract}

Keywords-cultural sovereignty; construction principles; selfrenewal

\section{INTRODUCTION}

With the development of new rural construction, new rural areas with unique cultural connotation all over the country have been completed. Within the current planning limited area, infrastructure construction and other constraints, rural dwellings form buildings with various representative symbolic such as Hui, Minnan, and courtyard and so on, on the basis of original dwellings forms [1]. We all stress that they inherited the original local architectural form with local characteristics, and through this shape that is different from the existing urban conglomerates, promote the development of tourism to support local farmers' employment. When the modern people in the praise of the town of the appropriate scale, architectural style, and roadway space, these preserved town was only one of the country gathered in the country then. When the future after several years if some of the current gathering of residential areas are retained, how will the future think of the current residential buildings and communities? What are the meanings of keeping some of the modern houses for the future? What kind of characteristics of modern houses can be retained?

\section{A. Characteristics of Rural Dwellings}

The content of rural dwellings includes two aspects, first, residential; Second, the living environment of residential extension.

The construction principle of rural dwellings is divided into three aspects: the comprehensive performance of the price and quality, such as local mature construction technology, durable materials, reasonable structures, and forms based on the functional; Second, on the basis of cost-effective, rural dwellings follow the local physical geography, the construction of the law at that time, and the customary cultural characteristics [2]. Three on the basis of the above two, rural dwellings pursuit architectural metaphor, such as order, humanities, scenes, personal spirit and material and so on.

\section{B. The current status quo}

With the process of urbanization, the original mode of residence has undergone great changes, residential houses surrounding the city was included in the territory of the city in city expansion, being demolished. Residential house that has not been included in the city territory also undergone reconstruction and renovation due to the use of their own lives

In this process, the urban landscape reconstruction part is basically a market - oriented commercial housing, designed by the design company and built by developer, operating in accordance with the laws of the market.

The self-reconstruction and renovation part of rural dwellings mostly changed in original construction methods and materials. Based on first cost-effective choice, residential houses are using the same materials, technology, and structure throughout the country. In the absence of a unified law on formation, residential houses are also built on the builder's own aesthetic taste. Although chaos, this is a phenomenon should not be accused, for the construction of modern houses still follows the above principles with the contents of this principle having been changed a little in the moment. This is because of the common effects of our market-oriented guidance, staff mobility, explosive number, cultural openness, and the ignorance of the traditional residential metaphor. Only when the results found to bring homogenization and chaos would current construction system and construction principles began to be reflected [3].

\section{Home and abroad}

In contrast to the status quo at home and abroad, example of foreign protection of the results and policies will always be analyzed. But the current promotion of ecological design, less intervention design, self-renewal and other reference, in fact, has already been in the traditional Chinese residential construction principles. Based on cost-effective principle, traditional houses will use the local materials according to local conditions so that in the period logistics is not developed, the main building materials are from the local. Naturally, the construction of these materials will be consistent with the surrounding environment. But today, with large-scale materials, marketization, and convenient transportation, the local materials and processes become customized and scarce. 
In the contained meaning of construction, the builders of traditional houses have awe of nature, and they feel that some of the natural objects such as mountains, water and trees can provide shelter for mankind, so that they should be in awe and provide protection. With the fear of nature, the traditional Chinese houses express self-modesty, and the coexistence attitude with natural and other species, which inevitably requires self-discipline, respect living space for other species without emphasizing people-oriented nature. These spiritual demands lead to thinner and leaking features of the modeling. This kind of thin, cold modeling is a very good interpretation of the principle of self-discipline. Longing for natural also calls for the inevitable introduction of light, wind, plants and other elements and the construction of coordination. Therefore, the relationship between man and nature in the construction of traditional Chinese dwellings, the respect for ecology, and the self-discipline in contradiction with environment are in line with the trend of future world development and meet the longterm goal of long-term coexistence of mankind and the earth.

In the construction technology, due to China's long-term household registration management system, the mobility of personnel is limited. Local workers are the main labor force of the construction of local dwellings. At the same time, architectural design in the case of productivity is difficult to spread to residential construction. Besides, residential construction technology follows a unified French-style construction, greatly improving the construction of the aging. The uniformity of the program makes it possible to build the assembly buildings, which greatly saves the material, labor, and time. These efficient construction techniques are also coincident with the development direction today that China is promoting the development of large-scale assembly.

In summary, from the essence, China's residential construction principles are advanced, self-hematopoietic, and self-improved. But why is the current residential construction such a mess? Behind it is cultural identity of our people and self-confidence in future development, which are actually embodiments of national comprehensive strength. At present, China is on the right road of development. But due to late start, we have a certain gap with developed countries, leading to the recession of national cultural influence [4].

The protection and development of residential issues is actually the confrontation of a unique national cultural sovereignty and the increasingly convergence of a single world cultural sovereignty. Based on this concern, in 1972 the United Nations Educational, Scientific and Cultural Organization adopted the Convention on the Protection of the World Cultural and Natural Heritage. But with the integration of the world economy, especially after entering the era of science and technology, the weakening of cultural sovereignty are becoming more and more obvious. Except some of the unique conditions of the legacy of cultural relics are preserved, other cultural heritage are homogenized, for the protection of the current sovereign culture requires the active promotion of external forces, rather than based on their own hematopoietic function of self-renewal. With the strengthening of our national strength, people's living standards arise, the revival of residential culture will definitely usher in

\section{THE SELF-RENEWAL OF RURAL DWELLINGS}

So with the improvement of national strength, how would residential renew?

\section{A. Quality improvement}

On the design principles of the modern residential upgrade, renovation, and reconstruction, the improvement of quality should be placed in the first place.

The current status of quality improvement of the our country still remain in the stage of the decoration of the building, expressing in the more durable, more clean, and more rare materials, more symbolic luxury decoration, or a unified style of decoration and so on.

However, the real quality of the building has been neglected. The improvement of building quality refers to: First, to meet people's require of comfortable indoor life at the same time of zero construction consumption, which needs to use advanced environmentally friendly materials and technology, such as waterproof and anti-disease isolation materials, heat loss prevention of doors and windows materials, geothermal, solar energy use, recyclable energy recycling, and ultimately to ensure that building users safe and comfortable; Second, whether active or passive construction requires the minimum energy consumption to achieve the construction of the indoor environment - pleasant air, humidity, and temperature; Third, the building and the environment should have a good interactive complementary relationship, such as reducing the construction of land degradation hazards, the return of groundwater hazards, and generated self-purification of building garbage.

\section{B. The cost-effective choice}

Cost-effective upgrade is to build on the basis of the concept of quality improvement, or it's still just a pile of high energy consumption house without the premise of self-renewal that will be replaced in next round of choice. But the price and quality is still the market decision. In the current market, zero energy consumption building materials and technology, as well as the overall cost of the process is still high, people's income level can't support comprehensive, extensive use of these technologies, which are in need for policy and guidance support.

At the same time, local materials and the use of technology will be replaced by market convergence, because in the case of well-developed logistics, large-scale production caused a great impact on local small-scale manufacturing. Under this inevitable law of the market, we should also explore the operation of the local material process within the market.

Also, there should also be greater efforts to explore the recycling of materials. For example, $60 \%$ of modern building materials is concrete, which as the application of such a wide range of cheap materials but with low added value. Based on the future trend of the environmental design product and customization, we should process artfully the concrete materials, and produce custom-oriented products, promoting the material as a cheap and widely used material with cultural characteristics. Innovation of artistic use can improve the 
material's added value, and through the research of art of recycled concrete materials, we can enhance its ecological value.

\section{The art from User's perspective}

Why should we emphasize the art from the user's perspective? It is to distinguish the artistic nature of the dwellings other than the perspective of the city designer. China's residential construction is with no designer but built under the master-apprentice model. But why do we find it with art value? The reality lies in the construction of the principle of residential itself. When you comply with certain design principles, it will inevitably bring the corresponding artistic experience and at the same time optimize the best protection of future users and local cultural characteristics in the update process.

\section{The SigNIFICANCE OF MODERN DWELLINGS TO FUTURE DWELLINGS}

When we analyze the significance of modern dwellings to future dwellings, we divide it into three parts.

First, the future of residential design;

Second, the characteristics of current houses that could be retained;

Third, the meaning of reserving of contemporary houses on the future people.

\section{A. Future residential design}

Function: With the development of science and technology, the way people interact will change greatly, people's functional requirements for the residential will tend to only meet the requirements of full privacy. Party hall and living room will become a public space that need to be arranged several in one gathering point according to the number of people fitness such a function [5], centralized used and under unified management, as the current joint office. There will also be joint living room or gym. But people would not necessarily require smaller residential area, people will add features like private courtyard into the residential area.

Architectures: First, zero energy consumption, recyclable and integration are the basic technical requirements for future residential buildings; second, residential areas are not just to meet living requirements, especially in the future people are increasingly dependent on science and technology. The necessary decoration will be weakened, and the significance of the building itself will be more prominent. People will emphasize more on residential architecture scene and the meaning of the building itself. Again, regardless of how economy or technology will strengthen human integration and assimilation, different geographical characteristics and cultural heritage will result in the diversity of residential buildings.

Community: In view of the future development of science and technology, people's information will be transparent, therefore people will be more inclined to have completely isolated private space while more open, diverse gathering space. There will be more public space in community.
Different from the present basic layout of only roadway in addition to house, there will be a lot of semi-outdoor, outdoor and public indoor space. Future community relationships will be richer and more interesting..

\section{B. Current dwelling features that could be contained}

To explore the modern dwellings in the eyes of the future, we have to consider what kinds of modern dwellings or dwelling communities may be kept to the future.

First, it should residential community being able to face the future industrial restructuring and adjust itself (excluding other uncontrollable factors). In the updating process, these residential communities continue to self-renewal, not entirely relying on tourism development, but changing and constantly adjust their own regional industrial structure of the community in different times. When it regards to building, single building like Safu villa which with time, architecture, technology, and artistic significance will be preserved.

Second, community with a certain local cultural heritage and following the local geographical characteristics, local materials and technology will continue to develop. In the development process, these communities will inevitably face a lot of choices, usually economic factors. So they need certain investment and input, that is, in addition to relying on tourism revenue, it also needs local policy support and guidance, especially the local material technology market support if they are to retain

\section{The meaning of reserving contemporary houses on future people}

The reason why such a house can be retained lies in that in the future people's lifestyles will be greatly changed, like from stone era to iron era, from iron era to steam era, and then from steam era to technology era. Each change in the tool will be accompanied by changes in society, including people's way of life. Technology use will make people's lives integrated and transparent [6]. Resources and ecology will also make people more economical. These community layout which come from the past, closed, extended and free self-disciplined, along with the materials and technology with distinguished characteristics, can well reflect that residential communities with better relationship between people and nature will be retained, and rural dwellings will be the supplements of the future integration. More importantly, these residences which could self-adjust in industrial structure and constantly adapt to change is expression of speaking right of the owner of the residential houses.

\section{CONCLUSION}

This paper attempts to analyze the present situation and the future development of the rural dwellings and find a sustainable way of development of the rural dwellings with people and the earth living harmoniously, promoting the protection and development of residential houses to have their own hematopoietic function which is not only the imitation of the ancient houses, nor is it similar to the protection of endangered species. Many of the ideas are not data supported, but are based on the writer's own thinking, which is the 
shortcomings of this article. This is for relevant persons to exchange and make corrections.

\section{REFERENCES}

[1] Douglas Hofstadter, Godel, Escher, Bach : An Eternal Golden Braid. JSBN7-100-01323-2/B.188 Commercial Press published 1996.08

[2] WANG Zigeng, 《A Beautiful CountryTrilogy the representation and narrative of globalization infrastructure issues $》$ Time+Architecture 2016.02
[3] Tong Ming, Dong Yugan, Ge Ming, garden and architecture.ISBN: 9787508464626, China WaterPower Press 2009.04

[4] (Japan) Kuma "ten house theory". ISBN: 7208077932, Shanghai People's Publishing House, 2008.05

[5] Chen, W. and H. J. H. Brouwers, 《Alkali binding in hydrated Portland cement paste 》 Cement and Concrete Research, 2010, (Accepted for publication).

[6] LuJune 《From opposite to harmony Village vs. city-----Research on "village within city" in booming China》 2014.8. 